\title{
Development of (125)Te synchrotron-radiation-based Mössbauer spectroscopy
}

\section{$\operatorname{AUTHOR(S):~}$}

Kurokuzu, Masayuki; Kitao, Shinji; Kobayashi, Yasuhiro; Saito, Makina; Masuda, Ryo; Mitsui, Takaya; Yoda, Yoshitaka; Seto, Makoto

\section{CITATION:}

Kurokuzu, Masayuki ...[et al]. Development of (125)Te synchrotron-radiation-based Mössbauer spectroscopy. Hyperfine Interactions 2014, 226(1-3): 687-691

\section{ISSUE DATE:}

2014-04

URL:

http://hdl.handle.net/2433/199667

\section{RIGHT:}

The final publication is available at Springer via http://dx.doi.org/10.1007/s10751-0130995-2.; This is not the published version. Please cite only the published version.; この論 文は出版社版でありません。引用の際には出版社版をご確認ご利用ください。 
Noname manuscript No.

(will be inserted by the editor)

\title{
Development of ${ }^{125}$ Te Synchrotron-Radiation-Based Mössbauer Spectroscopy
}

\author{
Masayuki Kurokuzu • Shinji Kitao • \\ Yasuhiro Kobayashi · Makina Saito . \\ Ryo Masuda . Takaya Mitsui · Yoshitaka \\ Yoda . Makoto Seto
}

Received: date / Accepted: date

\begin{abstract}
The Mössbauer spectra of the $35.49 \mathrm{keV}$ excited state of ${ }^{125} \mathrm{Te}$ were observed in the energy domain using synchrotron radiation for the first time. The obtained isomer shifts and Debye temperature agree with previous experiments. These results show that this method will be applicable to various compounds.
\end{abstract}

Keywords ${ }^{125}$ Te Mössbauer spectroscopy $\cdot$ synchrotron radiation

\section{Introduction}

The Mössbauer effect has been observed for approximately 100 nuclear transitions of nearly 50 elements. It has been used in various fields such as chemical, biological, earth, and fundamental physical sciences. However, except for some elements such as ${ }^{57} \mathrm{Fe},{ }^{119} \mathrm{Sn}$, and ${ }^{151} \mathrm{Eu}$, it is not easy to obtain a radioactive isotope source for each nuclear transition. Energy-tunable, highly-bright, and polarized synchrotron radiation (SR) offers an alternative and is an easily accessible choice as a source of Mössbauer spectroscopy. Usually, Mössbauer effect measurements using SR have been performed in the nuclear forward scattering that gives the time domain spectra. However, these spectra are sometimes difficult to analyze,

M. Kurokuzu · S. Kitao · Y. Kobayashi · R. Masuda · M. Seto

Research Reactor Institute, Kyoto University, Kumatori-cho, Sennan-gun, Osaka 590-0494, Japan

E-mail: kurokuzu@post3.rri.kyoto-u.ac.jp

T. Mitsui · M. Seto

Japan Atomic Energy Agency, 1-1-1 Koto, Sayo-cho, Sayo-gun, Hyogo 679-5148, Japan

Y. Yoda

Japan Synchrotron Radiation Research Institute, 1-1-1 Koto, Sayo-cho, Sayo-gun, Hyogo 6795198, Japan

M. Saito

Sincrotrone Trieste, SS 14 - km 163, 5 Basovizza, Trieste, Italy

M. Kurokuzu · S. Kitao · Y. Kobayashi · M. Saito · R. Masuda · T. Mitsui · Y. Yoda · M. Seto CREST, Japan Science and Technology Agency, Honcho Kawaguchi, Saitama 332-0012, Japan 
especially when the materials under study have multiple sites. Alternatively, energy domain methods using SR that afford absorption type Mössbauer spectra are similar to the spectra obtained with radioactive sources and complicated spectra are slightly easier to analyze[1]. Recently, the magnetic and vibration properties of $\mathrm{Te}$ in $\mathrm{Fe}_{1+y} \mathrm{Te}$ have been studied to elucidate the superconducting mechanism of iron chalcogenide superconductors[2]. and ${ }^{125}$ Te Mössbauer spectroscopy is a suitable technique for this study. We have developed a system for ${ }^{125}$ Te SR-based Mössbauer spectroscopy and show results confirming its feasibility for materials science research.

\section{Experiment}

${ }^{125}$ Te SR-based Mössbauer Spectroscopy was performed at the Nuclear Resonant Scattering beam line (BL09XU) of SPring-8. The storage ring was operated at the special timing mode (4/58-filling +53 bunches) and a ring current of 100 $\mathrm{mA}$ by top-up injections. This mode contained 53 isolated bunches, each interval was 82.6 ns, and a $4 / 58$-filled 2 -ns-spaced bunch train. The experimental setup is shown in Fig.1. A liquid-nitrogen cooled pre-monochromator was used to handle

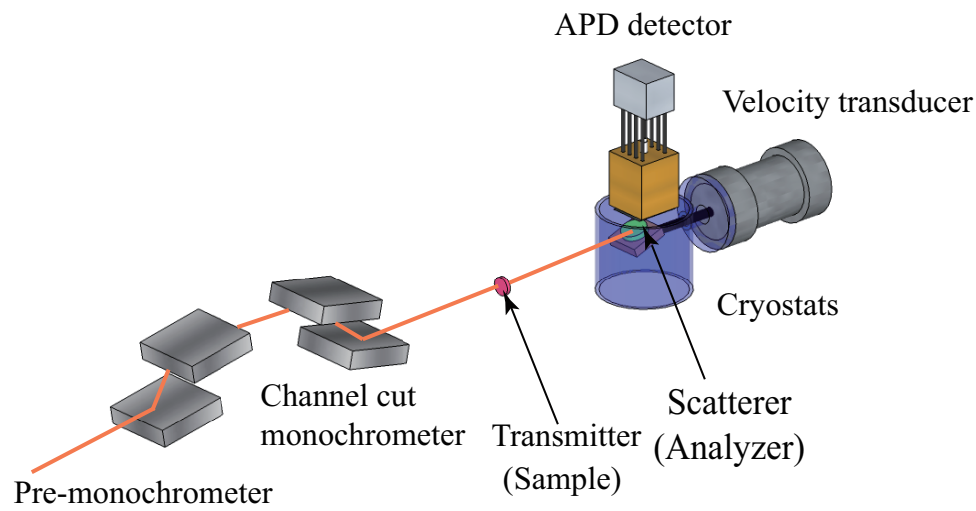

Fig. 1 Schematic of experimental setup. The glancing angle of the incident radiation with respect to the surface of the scatterer is approximately $7^{\circ}$ and delayed emission from the irradiated surface of the scatterer was measured using a multielement Si-APD detector $(4 \times 2$ APDs elements, with a detection area of $3 \times 5 \mathrm{~mm}^{2}$ each).

the high-heat load of the undulator radiation of SPring-8. An Si (1060) asymmetric channel-cut monochromator was then used to select SR with the nuclear resonance energy of $35.49 \mathrm{keV}$. For this method, two targets, a transmitter and a scatterer containing the same ${ }^{125} \mathrm{Te}$ Mössbauer nuclei are used; the scatterer is placed behind the transmitter on the path of the synchrotron beam. The scatterer was moved using a Mössbauer velocity transducer and was cooled by connecting it to the cold head of a He flow cryostat with $\mathrm{Cu}$ springs. The thickness of the springs was chosen to achieve a balance between the smooth motion of the scatterer and 
the cooling power. The delayed scattering from the scatterer was measured using a multielement Si-avalanche photodiode (APD) detector placed above the scatterer; wherein, the scatterer was inclined by approximately $7^{\circ}$ against the beam direction. It contains $4 \times 2$ arrays of APD elements with a detection area of 3 $\times 5 \mathrm{~mm}^{2}$ each. Fluorescent X-rays $\left(\mathrm{K}_{\alpha}: 27.4 \mathrm{keV}, \mathrm{K}_{\beta}: 31.0 \mathrm{keV}\right)$ accompanied by the de-excitation were detected, adding to the $\gamma$-rays directly emitted from the excited state $(35.49 \mathrm{keV})$. The detection efficiencies of the APD detector were estimated to be $0.05,0.04$, and 0.03 for $27.4,31.0$, and $35.49 \mathrm{keV} \mathrm{X}$-rays, respectively. The time resolution of the detector was around $1 \mathrm{~ns}$. The velocity-dependency of the scattering intensity yielded a spectrum in the energy domain. We used a Mössbauer calibrator consisting of a laser interferometer to determine the velocity of the transducer.

In the case of SR-based Mössbauer spectroscopy, a reference sample as either the scatterer or transmitter is a required addition to the target sample. We used ${ }^{125} \mathrm{Te}$-enriched $\mathrm{Mg}_{3} \mathrm{TeO}_{6}$ for both target and reference samples because it shows a single line Mössbauer absorption spectrum and has a high recoilless fraction even at room temperature[3]. The enriched $\mathrm{Mg}_{3} \mathrm{TeO}_{6}$ was synthesized by the solid reaction method described in Ref.[3]. To optimize the effective thickness, the enriched $\mathrm{Mg}_{3} \mathrm{TeO}_{6}$ powder was mixed with boron nitride and polyethylene powders and pressed to form pellets with a diameter of $10 \mathrm{~mm}$. The pellets with a thickness of $0.5 \mathrm{mg}{ }^{125} \mathrm{Te} / \mathrm{cm}^{2}$ and $5.0 \mathrm{mg}{ }^{125} \mathrm{Te} / \mathrm{cm}^{2}$ were used as the scatterer and the transmitter, respectively.

\section{Result and discussion}

A typical count rate of prompt electronic scattering was approximately $9.0 \times 10^{6}$ counts per second (cps). The delayed scattering signals within a time window from 8 to 14 ns were counted after each prompt signal, and it's typical count rate was approximately $3 \mathrm{cps}$. The ${ }^{125} \mathrm{Te}$ Mössbauer spectrum of the $\mathrm{Mg}_{3} \mathrm{TeO}_{6}$ transmitter at $300 \mathrm{~K}$ and the scatterer at $60 \mathrm{~K}$ is shown in Fig.2(a). The total acquisition time was about 8 hours. The spectrum showed a single line as reported in Ref.[3]. For simplicity, a Lorentzian line shape was used to fit the spectrum. The isomer shift obtained by fitting the observed spectrum is $0.1(2) \mathrm{mm} / \mathrm{s}$ and is consistent with the value of $0.01(2) \mathrm{mm} / \mathrm{s}$ obtained by conventional Mössbauer spectroscopy[3]. Although the difference of second-order Doppler shifts between 300 and $78 \mathrm{~K}$ is reported to be $0.01(2) \mathrm{mm} / \mathrm{s}[3]$, the apparent difference of the isomer shifts was within experimental errors and was not observed. Additionally, the ${ }^{125} \mathrm{Te}$ Mössbauer spectrum of $\mathrm{Mg}_{3} \mathrm{TeO}_{6}$ at $300 \mathrm{~K}$ with the scatterer at $300 \mathrm{~K}$ is shown in Fig.2(b). The total acquisition time was about 12 hours. The obtained isomer shift was $0.3(3) \mathrm{mm} / \mathrm{s}$ at $300 \mathrm{~K}$. This measurement proves that $\mathrm{Mg}_{3} \mathrm{TeO}_{6}$ can be used as the scatterer at room temperature and that it reduces the experimental difficulty by removing the cryostat apparatus of the scatterer. We also evaluated Debye temperature $\theta_{D}$ from the temperature dependence of the normalized absorption area in the spectra using the Debye model. Obtained $\theta_{D}$ was about $340 \mathrm{~K}$, and this value is consistent with the values obtained by conventional methods, 390(2) $\mathrm{K}[3]$ and $320 \mathrm{~K}[4]$.

Although we measured the ${ }^{125}$ Te Mössbauer spectra at the special timing mode (82.6 ns bunch interval) of the storage ring, appropriate bunch intervals, such as 


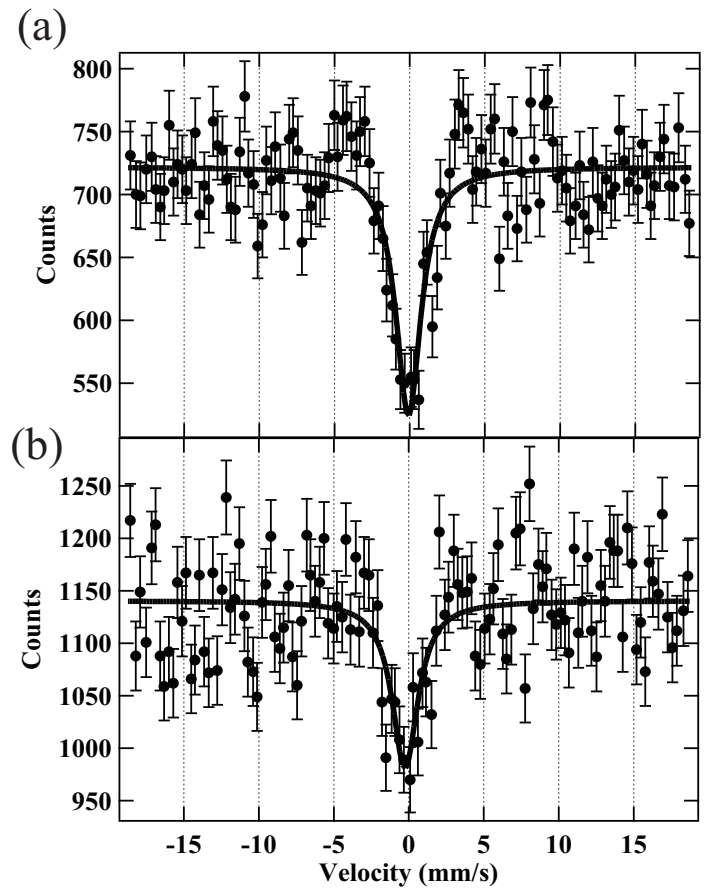

Fig. $2{ }^{125}$ Te Mössbauer spectra measured by using SR. As a transmitter, $\mathrm{Mg}_{3} \mathrm{TeO}_{6}$ with a thickness of $5.0 \mathrm{mg}{ }^{125} \mathrm{Te} / \mathrm{cm}^{2}$ was used at $300 \mathrm{~K}$. As a scatterer, $\mathrm{Mg}_{3} \mathrm{TeO}_{6}$ with a thickness of $0.5 \mathrm{mg}{ }^{125} \mathrm{Te} / \mathrm{cm}^{2}$ was used at (a) 60 and (b) $300 \mathrm{~K}$. The solid line shows fit spectra with Lorentzian line shapes.

the 203 bunch mode with 23.6 ns bunch intervals, improves the count rate because the half-life of the ${ }^{125} \mathrm{Te}(35.49 \mathrm{keV}$ first excited state) is $1.48 \mathrm{~ns}$. Furthermore, if the time resolution of the APD detector is improved, a measurement with an earlier timing gate after the prompt pulse is possible. For example, if the delayed scattering signals within a time window from 7 to $14 \mathrm{~ns}$ can be measured as in Ref.[1], we can obtain signal approximately 1.8 times larger than those from 8 to 14 ns. Finally, the detection of internal conversion electrons, well known in conventional Mössbauer spectroscopy and previously measured by nuclear resonance scattering of SR by ${ }^{197} \mathrm{Au}[5]$, is expected to substantially improve efficiency due to the internal conversion process, which is dominant in ${ }^{125} \mathrm{Te}$ (the internal conversion coefficient is 13.6). Therefore, ${ }^{125}$ Te SR-based Mössbauer spectroscopy is promising for many fields of investigations on various Te-containing compounds, including the superconductor $\mathrm{Fe}_{1+y} \mathrm{Te}$.

\section{Summary}

The ${ }^{125}$ Mössbauer spectra were measured using SR for the first time. It was found that $\mathrm{Mg}_{3} \mathrm{TeO}_{6}$ can be used as a scatterer at room temperaturefor ${ }^{125} \mathrm{Te}$ SRbased Mössbauer spectroscopy. The isomer shifts and $\theta_{D}$ of $\mathrm{Mg}_{3} \mathrm{TeO}_{6}$ obtained 
by SR-based Mössbauer spectroscopy are consistent with the values reported in conventional methods. These results show that this spectroscopic method can be applied to various Te-containing compounds. There are possibilities to enhance the effectiveness of this method through the optimization of bunch mode, improvement of time resolution in the APD detector, and detection of the internal conversion electrons.

Acknowledgements We thank the Accelerator Group of SPring- 8 for their efforts in obtaining pure bunches and the staff at SPring- 8 for their support. These experiments were performed at the BL09XU of SPring-8 with the approval of the Japan Synchrotron Radiation Research Institute (JASRI) (Proposal No. 2009B0086).

\section{References}

1. M. Seto, R. Masuda, S. Higashitaniguchi, S. Kitao, Y. Kobayashi, C. Inaba, T. Mitsui, Y. Yoda, Phys. Rev. Lett. 102(21), 217602 (2009)

2. F.C. Hsu, J.Y. Luo, K.W. Yeh, T.K. Chen, T.W. Huang, P.M. Wu, Y.C. Lee, Y.L. Huang, Y.Y. Chu, D.C. Yan, et al., Proc. Natl. Acad. Sci. USA 105(38), 14262 (2008)

3. W. Bresser, M. Zhang, L. Koudelka, J. Wells, P. Boolchand, G. Ehrhart, P. Miller, Phys. Rev. B 47(18), 11663 (1993)

4. M. Pasternak, M. Van der Heyden, G. Langouche, Nucl. Instrum. Methods, B 4(1), 152 (1984)

5. S. Kishimoto, Y. Yoda, M. Seto, Y. Kobayashi, S. Kitao, R. Haruki, T. Kawauchi, K. Fukutani, T. Okano, Phys. Rev. Lett. 85(9), 1831 (2000) 